\title{
Aggressive Typical Carcinoid Tumor of the Sphenoid Sinus with Uncommon Clinicopathological Presentation
}

\author{
Deok-Soo Kim ${ }^{1}$, Sung-Dong Kim ${ }^{1}$, Hak-jin Kim ${ }^{2}$ and Kyu-Sup Cho ${ }^{1,{ }^{*}}$ \\ ${ }^{1}$ Department of Otorhinolaryngology and Biomedical Research Institute, Pusan National University Hospital, Pusan National University School of Medicine, Pusan, South \\ Korea \\ ${ }^{2}$ Department of Radiology, Pusan National University Hospital, Pusan National University School of Medicine, Pusan, South Korea \\ "Corresponding author: Department of Otorhinolaryngology and Biomedical Research Institute, Pusan National University Hospital, Pusan National University School of \\ Medicine, Pusan, South Korea. Email: choks@pusan.ac.kr
}

Received 2018 August 26; Revised 2018 October 03; Accepted 2018 December 25.

\begin{abstract}
Carcinoid tumors are neuroendocrine tumors that usually occur in the digestive tract and lung. They are rarely found in the head and neck, especially in the sinonasal area. This article describes a typical carcinoid tumor involving the sphenoid sinus, cavernous sinus, sphenoid bone, clivus, and occipital condyle, which was treated successfully by endoscopic transseptal transsphenoidal surgery and intensity-modulated radiation therapy.
\end{abstract}

Keywords: Carcinoid Tumor, Neuroendocrine Tumors, Sphenoid Sinus, Radiotherapy, Intensity-Modulated

\section{Introduction}

Carcinoid tumors are rare and indolent neuroendocrine tumors (NETs). The majority occur in the gastrointestinal tract (54.4\%), followed in order of decreasing frequency, by the lung and bronchi (30.1\%), pancreas $(2.3 \%)$, ovary (1.2\%), biliary tract (1.1\%), and head and neck $(0.4 \%)$ (1). NETs are found very rarely in the nasal cavity and the paranasal sinuses, accounting for approximately $5 \%$ of sinonasal malignancies (2). However, there have been few reports of carcinoid tumors arising in the nasal cavity and paranasal sinuses, and no previous reports of aggressive typical carcinoid tumors originating in the sphenoid sinus. This case report describes a rare clinical presentation of typical carcinoid tumor involving the sphenoid sinus, cavernous sinus, sphenoid bone, clivus, and occipital condyle, which was treated successfully by endoscopic transseptal transsphenoidal surgery and intensitymodulated radiation therapy (IMRT).

\section{Case Presentation}

A 53-year-old woman was referred to our clinic with an incidentally found posterior nasal cavity mass lesion. She had experienced progressive bilateral nasal obstruction for 6 months. She had no relevant medical history and no other symptoms. Endoscopic examination showed a huge polypoid mass in the posterior nasal cavity between the right middle turbinate and nasal septum (Figure 1A). Paranasal sinus computed tomography(CT) demonstrated a solid mass in both sphenoid sinuses with osteolytic lesion in both sides of the body, greater wing, left pterygoid plate of sphenoid bone, clivus, and left occipital condyle (Figure $1 \mathrm{~B}$ and $\mathrm{C}$ ). On magnetic resonance (MR) imaging, a solid mass involving the left cavernous sinus with encasement of the intracavernous carotid artery had low signal intensity on T1-weighted images (T1WIs), heterogenous high signal intensity on T2WIs, and strong enhancement on gadolinium-T1WIs (Figure1D-F). We performed transseptal endoscopic removal under general anesthesia. Initially, conventional septoplasty was performed with hemitransfixion incision on the concave side. We partially removed the vomer and perpendicular plate of ethmoid to improve surgical visibility and accessibility to the sphenoid sinus. Both sphenoid ostia were opened and connected to each other. A mass lesion found in the sphenoid sinus was fragile and bled easily. Frozen biopsy suggested that this tumor was possibly a well-differentiated NET. The tumors were partially removed and histopathological examination revealed uniform cells with hyperchromatic nuclei. Mitotic figures were very rare, and no necrosis was identified. On immunohistochemical staining, the lesion was positive for synaptophysin, pancytokeratin, and CD56.

These findings were consistent with a typical carcinoid tumor (Figure 2). Positron emission tomography-CT per- 

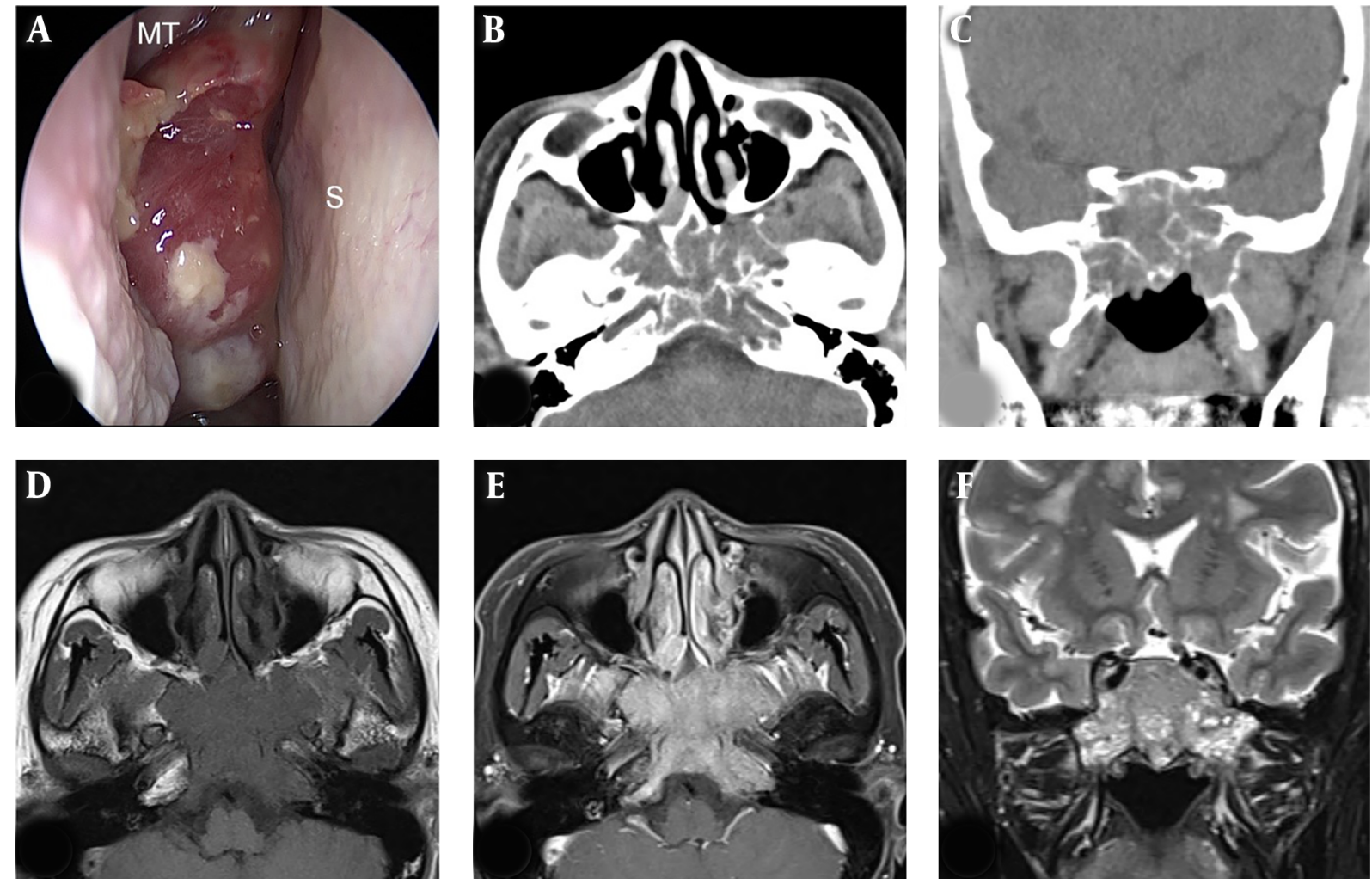

Figure 1. Preoperative endoscopic and radiological findings. Nasal endoscopy (A) showed a huge polypoid mass between the right middle turbinate (MT) and nasal septum (S). Axial (B) and coronal (C) images of computed tomography shows a solid mass in both sphenoid sinuses with osteolytic lesions on both sides of the body, greater wing, and left pterygoid plate of sphenoid bone, clivus, and left occipital condyle. The mass involving the left cavernous sinus with encasement of the intracavernous carotid artery showed low signal intensity on T1 axial (D), strong enhancement on post-contrast T1 axial (E), and heterogeneous high signal intensity on T2 coronal (F) magnetic resonance images.

formed 2 months postoperatively showed no primary recurrence or any other metastasis. The patient received IMRT at a dose of 50 Gy in 25 fractions over 6 weeks to eradicate any residual tumor. Recovery was uneventful and the patient's symptoms have disappeared. Endoscopic examination and MR imaging performed 2 years after radiotherapy demonstrated no aggravation of the preexisting tumor (Figure 3).

\section{Discussion}

Carcinoid tumors occur most commonly in the colon, rectum, and cecum, and are derived from the enterochromaffin cells of gastrointestinal and bronchopulmonary systems (3). Carcinoid tumors rarely develop in the head and neck, especially in the sinonasal area, as primary tumors. Furthermore, typical carcinoid tumors arising in the sphenoid sinus are extremely rare, and the present case is unique in that it presented as an aggressive typical carcinoid tumor involving the right nasal cavity, sphenoid bone, clivus, and occipital condyle.
The clinical features of this tumor were nonspecific and similar to those of other sinonasal tumors. Common presentations include epistaxis, nasal obstruction, exophthalmos, nasal pain, and headache (4). Although a typical carcinoid tumor is usually non-functional, it may cause the carcinoid syndrome (4). Flushing, diarrhea, and cardiac involvement are typical symptoms of the classic carcinoid triad (5). However, carcinoid syndrome occurs only in about $10 \%$ of patients with carcinoid tumors (5). In this case, the patient did not have any other symptoms of carcinoid syndrome.

Diagnosis is based on the characteristics of the carcinoid tumor on histopathological and immunohistochemical analyses for chromogranin A, synaptophysin, neuron specific enolase, CD56, and cytokeratins (4). Typical carcinoid tumors are well-differentiated lesions and are distinguished from moderately differentiated atypical carcinoid tumors by the absence of mitoses, pleomorphism, and necrosis (6). Typical carcinoids are less aggressive and are managed differently, making an accurate diagnosis is 

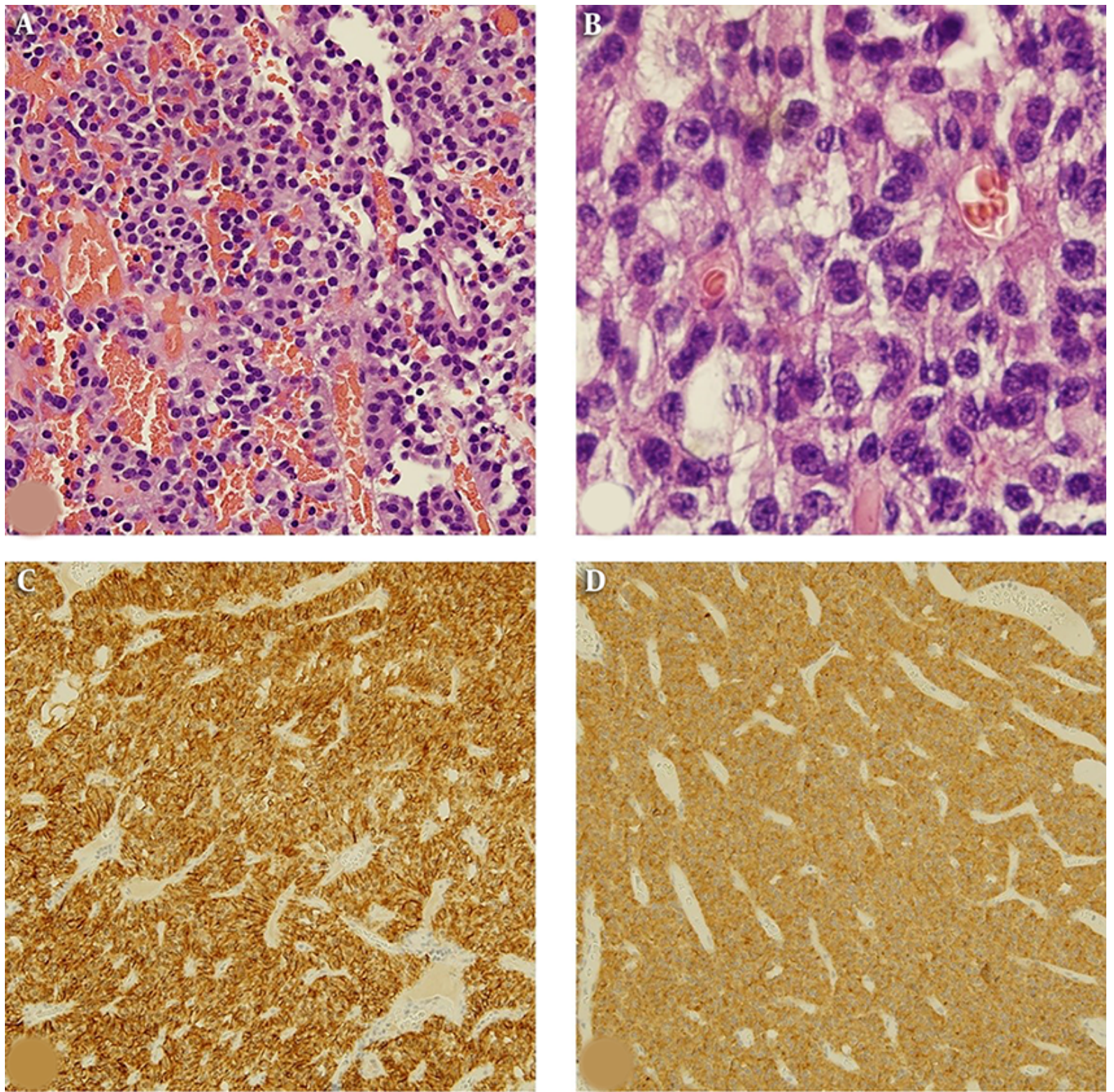

Figure 2. Histopathological findings of the typical carcinoid tumor. This tumor was composed of monomorphic tumor cells showing insular or trabecular growth pattern without necrosis and mitosis (hematoxylin and eosin, $\times 200$ ) (A). Tumor cells had finely granular cytoplasm and salt and pepper chromatin (hematoxylin and eosin, $\times 1000$ ) (B). Tumor cells were positive for CD56 (C) and synaptophysin (D) on immunohistochemistry

critical.

An optimal treatment method for typical carcinoid tumors of the paranasal sinuses has not been established because these lesions are very rare. Treatment is based on the location of the primary tumor and guided by whether metastasis has occurred. Although complete surgical resection has been the most successful treatment, radiotherapy and chemotherapy should be performed as postoperative adjuvant therapy when complete resection is difficult and surgery is ineffective (2). In the present case, total surgical resection of the typical carcinoid tumor of the sphenoid sinus was difficult because the tumor had invaded the sphenoid bone, clivus, and cavernous sinus. However, this tumor was a well-differentiated and low grade malignancy without involvement of neck nodes and distant sites, which was successfully treated by endoscopic transseptal transsphenoidal surgery and IMRT.

Although aggressive and destructive behavior of a typ- 

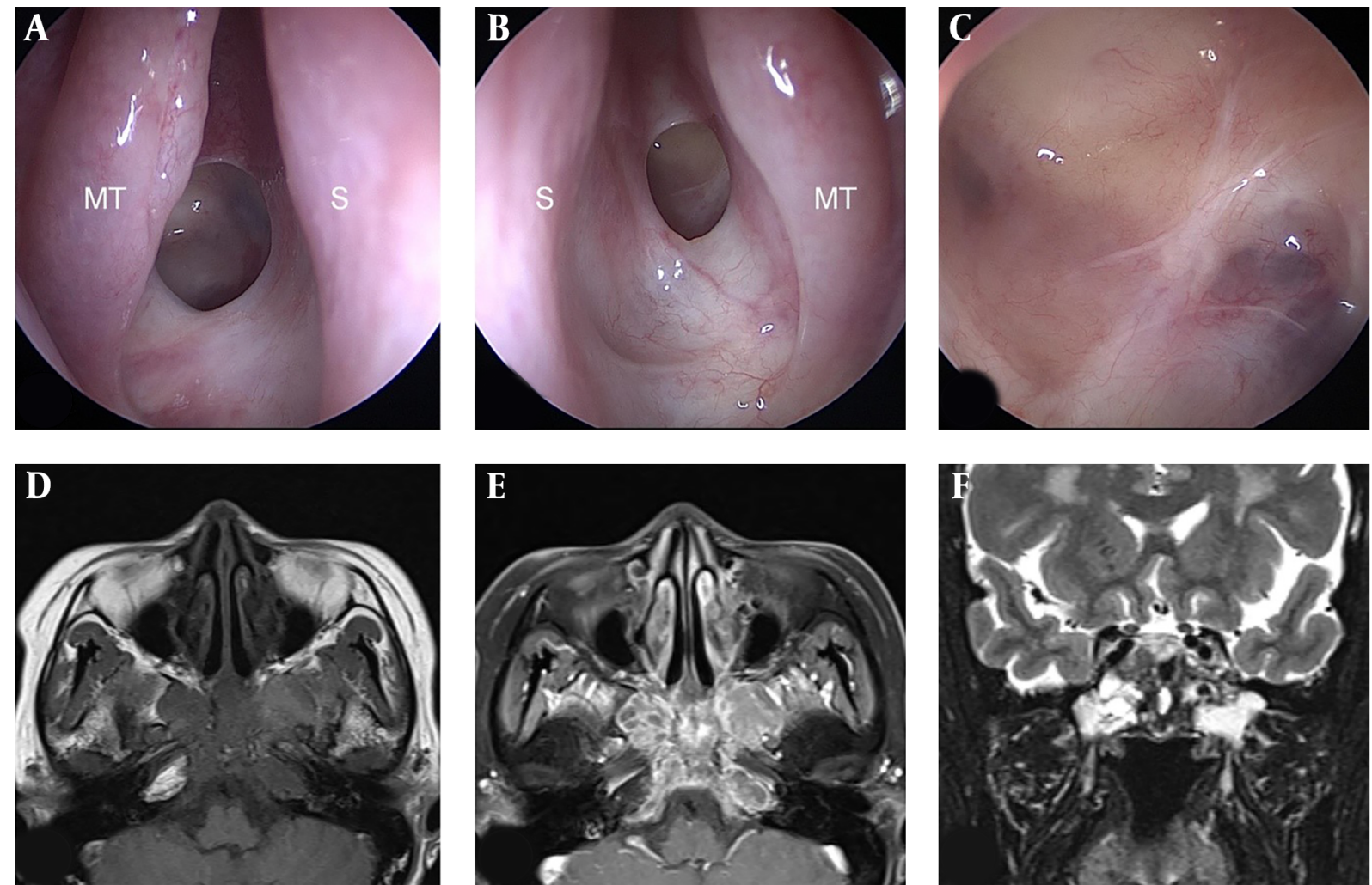

Figure 3. Endoscopic and magnetic resonance findings after surgery and radiotherapy. Nasal endoscopy showed no evidence of remnant tumor in the right nasal cavity (A), left nasal cavity (B), and the sphenoid sinus (C). Typical carcinoid tumor showed no significant interval change in size or signal intensity on T1 axial (D), post-contrast T1 axial (E), and T2 coronal (F) images. (MT, middle turbinate; $S$, nasal septum).

ical carcinoid tumor is very rare, it is important to consider this disease entity in the differential diagnosis of sinonasal tumor. When it appears impossible to completely resect a locally aggressive typical carcinoid tumor in the sphenoid sinus, radiotherapy could be considered as postoperative adjuvant therapy.

\section{Footnotes}

Authors' Contributions: All authors of this manuscript have directly participated in the planning, execution, or analysis of this study. Deok-Soo Kim: drafting the article,and revising it; Sung-Dong Kim: analysis and interpretation of data; Hak-Jin Kim: analysis and interpretation of image; Kyu-Sup Cho: drafting the article, conception and design

Conflict of Interests: None of the authors has any conflict of interest.

Financial Disclosure: None declared.

Funding/Support: None of the authors has any financial or funding support.

\section{References}

1. Maggard MA, O'Connell JB, Ko CY. Updated population-based review of carcinoid tumors. Ann Surg. 2004;240(1):117-22. [PubMed: 15213627]. [PubMed Central: PMC1356383].

2. Renner G. Small cell carcinoma of the head and neck: A review. Semin Oncol. 2007;34(1):3-14. doi: 10.1053/j.seminoncol.2006.10.024. [PubMed: 17270660].

3. Modlin IM, Lye KD, Kidd M. A 5-decade analysis of 13,715 carcinoid tumors. Cancer. 2003;97(4):934-59. doi: 10.1002/cncr.11105. [PubMed: 12569593].

4. Mitchell EH, Diaz A, Yilmaz T, Roberts D, Levine N, DeMonte F, et al. Multimodality treatment for sinonasal neuroendocrine carcinoma. Head Neck. 2012;34(10):1372-6. doi: 10.1002/hed.21940. [PubMed: 22052583].

5. Modlin IM, Oberg K, Chung DC, Jensen RT, de Herder WW, Thakker RV, et al. Gastroenteropancreatic neuroendocrine tumours. Lancet Oncol. 2008;9(1):61-72. doi: 10.1016/S1470-2045(07)70410-2. [PubMed: 18177818].

6. Lee $\mathrm{DH}, \mathrm{Cho} \mathrm{HH}, \mathrm{Cho}$ YB. Typical carcinoid tumor of the nasal cavity.Auris Nasus Larynx. 2007;34(4):537-9. doi:10.1016/j.anl.2007.02.010. [PubMed: 17481837]. 